\title{
Mariguana y sus efectos sobre el cerebro, la toma de decisiones y la inteligencia. Una revisión narrativa
}

\author{
Alejandra E. Ruiz-Contreras', Ivett Ortega-Mora', Octavio Amancio Belmont² , Mónica Méndez-Díaz², Óscar \\ Prospéro-García ${ }^{2}$ \\ Grupo Neurociencias: Lab. Neurogenómica Cognitiva, Coord. Psicobiología y Neurociencias, Fac. de Psicología, Universidad Nacional Autónoma de \\ México, México \\ ${ }^{2}$ Grupo Neurociencias: Lab. Cannabinoides, Depto. de Fisiología, Fac. de Medicina, Universidad Nacional Autónoma de México, México
}

\section{RESUMEN}

Introducción: la mariguana es la droga de mayor consumo después del tabaco y el alcohol, a pesar de que está penado utilizar más de cierto gramaje que depende del país; por ejemplo, en México es más de $5 \mathrm{~g}$, lo que indica una alta motivación por conseguir la droga. Uno de los principios activos de la mariguana es el $\Delta 9$-tetrahidrocannabinol. Esta molécula afecta al sistema endocannabinoide, provocando cambios en su función y, concomitantemente, en la morfología cerebral. Objetivo: revisar y discutir la evidencia científica del efecto del consumo de mariguana sobre el cerebro, la inteligencia y la toma de decisiones. Método: se consideraron los reportes científicos que abarcan de 2005 a marzo de 2016 encontrados en PubMed, enfocados en los efectos del consumo de mariguana sobre cambios morfológicos cerebrales, la inteligencia y la toma de decisiones. Resultados: dependiendo de la edad de inicio del consumo de mariguana, es posible detectar cambios en el cerebro y la inteligencia, mientras que otras variables como la frecuencia o la cantidad de uso repercuten en la eficiencia para la toma de decisiones. Discusión y conclusiones: estos resultados señalan que 1) el consumo de mariguana afecta la morfología cerebral, la toma de decisiones y la inteligencia; 2) sugieren la potencial participación del sistema endocannabinoide en la toma de decisiones e inteligencia; y 3) dadas las consecuencias, deben alertar al sector salud para promover su prevención y en su momento estar preparados para la rehabilitación.

Palabras clave: mariguana, inteligencia, adolescencia, sistema endocannabinoide, toma de decisiones.

\begin{abstract}
Introduction: marijuana or cannabis is the most consumed drug after tobacco and alcohol, even in the condition that to carry more than a given amount is a crime, suggesting that cannabis consumers are highly motivated to get the drug. In Mexico the amount allowed is $5 \mathrm{~g}$. Delta-9-tetrahydrocannabinol is one of the major psychoactive ingredient of cannabis. This molecule acts upon the endocannabinoid system, producing modifications in their function and, consequently, in the brain morphology. Objective: to review and discuss the scientific literature about cannabis consumption effect on brain, intelligence and decision-making. Method: the search of scientific reports in PubMed from January, 2005 to March, 2016, to evaluate the marijuana effect on brain morphology, intelligence and decision making. Results: it seems that as a function of the age of cannabis consumption onset the user might have morphological and functional brain changes, whereas variables such as frequency or amount of cannabis use may affect the efficiency of decision making. Discussion and conclusions: all these data together indicate that: 1) marijuana consumption affects brain morphology, decision-making and intelligence; 2) there is a potential role of the endocannabinoid system in decision-making and intelligence; and 3) given these consequences, should alert health organizations to develop strategies aiming prevention and if it is the case rehabilitation.
\end{abstract}

Keywords: cannabis, intelligence, adolescence, endocannabinoid system, decision making.

\footnotetext{
Autor de correspondencia:

Dr. Óscar Prospéro-García. Departamento de Fisiología, Facultad de Medicina, Universidad Nacional Autónoma de México. Apartado postal 70-250, C.P. 04510, Ciudad de México. Tel. +52 (55) 562325 09. Correo electrónico: opg@unam.mx Recibido: 12 de abril de 2016 Aceptado: 7 de junio de 2016 DOI: 10.28931/riiad.2016.2.06
} 


\section{INTRODUCCIÓN}

De acuerdo con el World Drug Report 2015, la prevalencia anual del consumo de mariguana o cannabis es de 181.79 millones de personas, equivalente a $3.9 \%$ de la población mundial (Oficina de las Naciones Unidas contra la Droga y el Delito [UNODC, por sus siglas en inglés], 2015), mientras que el consumo de cocaína y opioides tiene una prevalencia del $0.4 \%$ para cada uno (UNODC, 2015). Esto señala a la mariguana como la droga ilegal más consumida, sólo después del tabaco y el alcohol.

Uno de los mitos entre la población consumidora es que la mariguana no provoca ningún tipo de daño ni dependencia. Sin embargo, es a partir de la quinta edición del Diagnostic and Statistical Manual of Mental Disorders (DSM-V) que se reconoce que el consumo de mariguana provoca dependencia, que se manifiesta por la presencia de tolerancia a sus efectos con el uso repetido a las mismas dosis, la falla en el control de su consumo, el deseo intenso por consumirla (craving) y la presencia de síndrome de abstinencia (American Psychiatric Association [APA], 2013; Haney, Ward, Comer, Foltin, \& Fischman, 1999a, 1999b). En este sentido, es relevante comunicar al sector salud las consecuencias del consumo de mariguana para prevenirlo y promover la rehabilitación.

La mariguana contiene más de 400 componentes, de los cuales 66 moléculas son cannabinoides (Huestis, 2005); entre ellos está el $\Delta 9$-tetrahidrocannabinol ( $\Delta 9$ $\mathrm{THC}$ ), el componente más estudiado, que produce los efectos psicoactivos reforzantes en el individuo: v. gr. sensación de bienestar y de "high" y cambios en la percepción. Además, se ha reportado que es el responsable del deterioro de la atención y la memoria, así como del aumento de la frecuencia cardiaca que el usuario prefiere no tener (Bossong et al., 2012; D'Souza et al., 2012; Hart, Van Gorp, Haney, Foltin, \& Fischman, 2001). El $\triangle 9$-THC ejerce su acción principalmente sobre el sistema endocannabinoide (SECB), actuando sobre diversos receptores como el receptor a cannabinoides 1 (CB1) y el receptor a cannabinoides 2 (CB2); pero también tiene acción sobre otros receptores, v.gr. el receptor acoplado a proteína G 55 (GPR55) y el receptor a vaniloides 1 (TRPV1).

Un efecto que provoca que el consumidor crea que la mariguana no provoca dependencia es que el $\triangle 9$-THC es secuestrado por el tejido adiposo y se libera lentamente por dos y hasta tres semanas, enmascarando el síndrome de abstinencia. Una vez que un individuo consume mariguana, $v$. gr., entre 10 y $15 \mathrm{mg}$ de $\Delta 9$-THC [un carrujo de mariguana puede tener alrededor de 10-400 mg de $\triangle 9$-THC (Ashton, 2001)], la concentración en plasma alcanza un pico máximo de $100 \mathrm{mcg} / \mathrm{L}$, cinco a siete minutos después. En el Sistema Nervioso Central (SNC) la concentración máxima es similar en áreas corticales (como en las cortezas frontal y occipital), así como en el cerebelo; mientras que en la médula oblongada tiene una concentración menor (Brunet et al., 2006). Las áreas corticales a las que rápidamente afecta el $\triangle 9-\mathrm{THC}$ son, en gran medida, áreas involucradas en la función cognitiva. A continuación, daremos una breve introducción sobre el sECB.

\section{Sistema endocannabinoide}

Está compuesto por ligandos endógenos: 2-araquidonilglicerol (2-AG), anandamida (AEA), oleamida (ODA; ver Tabla 1), enzimas de síntesis: ANA por la N-acil fosfatidil etanolamina-fosfolipasa D (NAPE-PLD) y el 2-AG por la diacilglicerol lipasa (DAG-L), enzimas de degradación: amidohidrolasa de los ácidos grasos (FAAH) y la monoacil glicerol lipasa (MAGL) y los receptores a cannabinoides 1 (CB1) y 2 (CB2). Estos receptores están expresados en tejidos periféricos (testículos, bazo, sistema inmunológico, sistema nervioso periférico), y el SNC (Howlett et al., 2002). El receptor CB1 está altamente expresado en el SNC (Burns et al., 2007; Ceccarini et al., 2015; Felder et al., 1996; Herkenman et al., 1991; Herkenham et al., 1990); está compuesto por 472 aminoácidos en el humano, es un receptor metabotrópico acoplado a una proteína G inhibidora (PGi) expresado en la presinápsis. Los endocannabinoides son liberados de la neurona postsináptica y activan al receptor CB1; éstos son sintetizados a consecuencia de la estimulación de la neurona postsináptica por la neurona presináptica, mediante algún neurotransmisor excitador, esto es, glutamato (Figura 1). La entrada de calcio a la neurona postsináptica a consecuencia de la estimulación excitadora activa a las enzimas de síntesis. De esta manera, se considera que los endocannabinoides se sintetizan a demanda. Una vez sintetizados, difunden al espacio sináptico y alcanzan al receptor CB1. La estimulación de este receptor inhibe la actividad de adenilato ciclasa $y$, en consecuencia, la síntesis de AMP cíclico. Asimismo, inhibe a los canales de calcio y activa a los canales de potasio hiperpolarizando a la membrana presináptica. Dichos efectos conducen a una disminución en la probabilidad de liberar el neurotransmisor de la neurona presináptica (Howlett et al., 2002; Méndez-Díaz, Herrera-Solís, Soria-Gómez, Rueda-Orozco, \& Prospéro-García, 2008).

La activación del receptor CB1 modula diversas funciones como el sueño (Herrera-Solís, Vásquez, \& Prospéro-García, 2010; Mendelson \& Basile, 1999; Méndez-Díaz et al., 2013), la ingestión de alimento (Kirkham, Williams, Fezza, \& Di Marzo, 2002; Soria-Gómez et al., 2007), el sistema de la motivación recompensa (Méndez-Díaz, 
Tabla 1

Cannabinoides con afinidad al receptor CB1

\begin{tabular}{|c|c|c|}
\hline $\begin{array}{c}\text { Tipo de } \\
\text { cannabinoide }\end{array}$ & Nombre & $\begin{array}{c}\text { Propiedades funcionales } \\
\text { sobre el CB1 }\end{array}$ \\
\hline \multirow[t]{7}{*}{ Endocannabinoides } & 2 araquidonil glicerol & Agonista completo \\
\hline & Anandamida & Agonista parcial \\
\hline & Oleamida & Agonista parcial \\
\hline & Noladin eter & Agonista selectivo \\
\hline & Homo-gamma-linoleniletanolamida & Agonista parcial \\
\hline & $\mathrm{N}$-araquidonil dopamina & Agonista \\
\hline & Virodamina & Antagonista \\
\hline \multirow[t]{3}{*}{ Fitocannabinoides } & $\Delta 9$-tetrahidrocannabinol & Agonnista selectivo \\
\hline & Cannabinol & Agonista débil \\
\hline & Cannabidiol & $\begin{array}{l}\text { Agonista muy débil Termina siendo } \\
\text { antagonista }\end{array}$ \\
\hline Cannabinoides & $\mathrm{HU}-210$ & Agonista completo \\
\hline \multirow[t]{8}{*}{ sintéticos } & CP-55,940 & Agonista completo \\
\hline & WIN 55,212-2 & Agonista selectivo \\
\hline & Nabilona & Agonista selectivo \\
\hline & Metanandamida & Agonista selectivo \\
\hline & ACEA & Agonista selectivo \\
\hline & AM-251 & Antagonista selectivo \\
\hline & AM-281 & Antagonista selectivo \\
\hline & SR141716A o Rimonabant & Antagonista selectivo \\
\hline
\end{tabular}

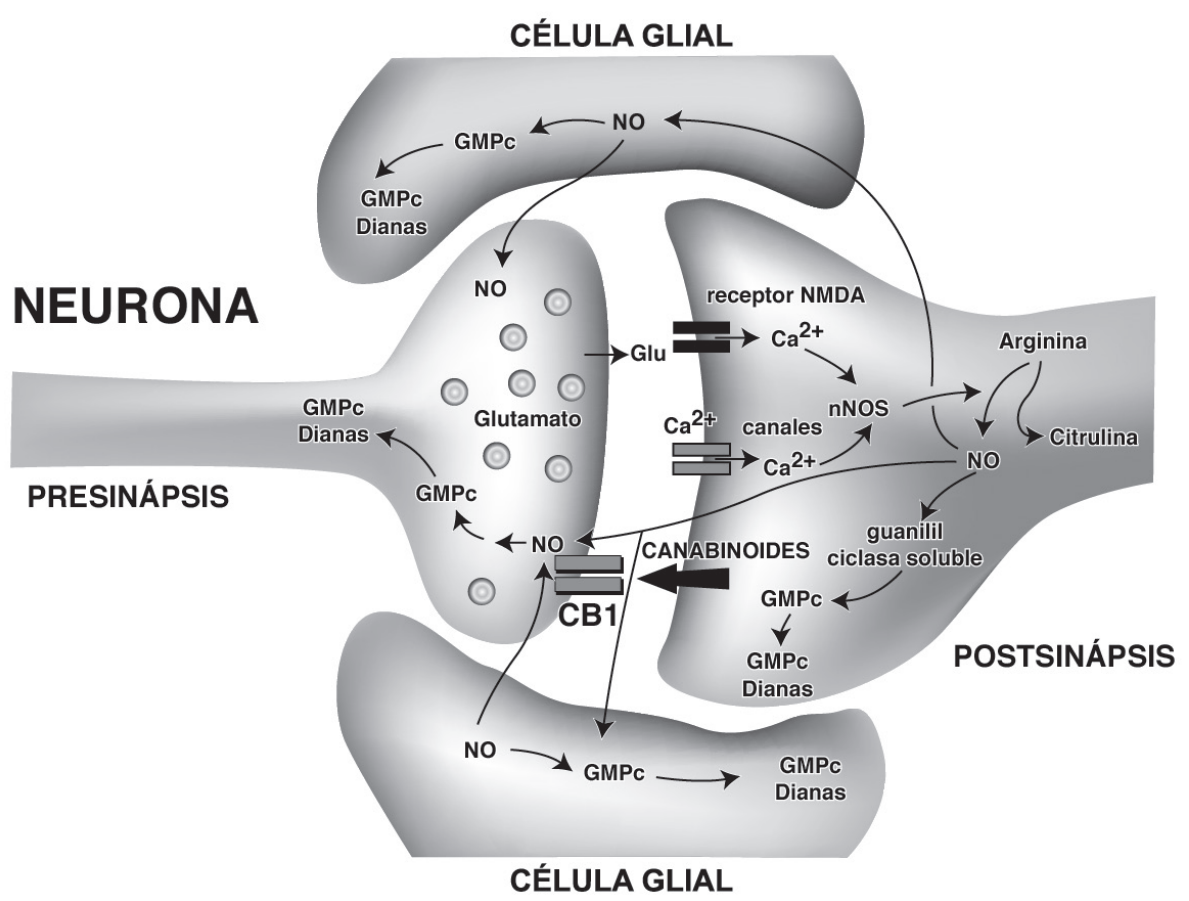

Figura 1. Sinápsis excitadora (glutamatérgica). Se ilustran los efectos del glutamato sobre una neurona postsináptica y los consecuentes cambios que amplifican la excitación, como es la formación de óxido nítrico y la formación de endocannabinoides, que reduce la liberación del glutamato y reduce la excitación de esta sinápsis 
Rueda-Orozco, Ruiz-Contreras, \& Prospéro-García, 2012; Romano-López, Méndez-Díaz, Ruiz-Contreras, Carrisoza, \& Prospéro-García, 2012), la memoria (Méndez-Díaz et al., 2008; Pedroza-Llinás, Méndez-Díaz, Ruiz-Contreras, \& Prospéro-García, 2013; Ruiz-Contreras et al., 2011) y la atención (Ruiz-Contreras et al., 2014). Dada su distribución en áreas asociadas con la cognición, el receptor CB1 es de interés para esta revisión.

Para poder estudiar los efectos del consumo de mariguana en la conducta es necesario considerar que hay otras diversas variables que pueden tener un impacto en la misma. Por ejemplo, la edad de inicio, el último consumo antes de evaluar al sujeto, si fue reciente o no, y el consumo concomitante de otras drogas de abuso. El tipo de mariguana, para estimar la potencial cantidad de THC que contenga y las potenciales sustancias agregadas a la misma, por ejemplo insecticidas, contaminantes, entre otras. Hacia los años 60 y 70 se reconocía que un cigarrillo de mariguana contenía entre 1 y $3 \%$ de $\Delta 9-\mathrm{THC}$, equivalente a alrededor de $10 \mathrm{mg}$; mientras que entre los años 80 y 90 este contenido aumentó entre 6 y 20\% de $\triangle 9$-THC, equivalente a 60-200 mg (Ashton, 2001). Actualmente hay otras variedades de mariguana como la $Y$ Griega, que puede tener más de $30 \%$, lo que sugiere tener entre 300 y $400 \mathrm{mg}$ de $\Delta 9$-THC. La concentración en plasma de un sujeto que ha fumado entre 10 y $15 \mathrm{mg}$ de $\triangle 9$-THC se calcula que es de $318 \mathrm{pmol} / \mathrm{ml}$, mientras que la concentración de anandamida (un endocanabinoide) es de $0.42 \mathrm{pmol} / \mathrm{ml}$ (Giuffrida et al., 2004). Es decir, casi mil veces más. Esto es, la concentración de $\triangle 9$-THC que estimula al CB1 supera por mucho a los ligandos endógenos, por lo que es de esperarse que el consumo de mariguana afecte la función del sistema endógeno.

Debido al interés actual de legalizar la mariguana para su uso medicinal y recreativo, resulta imprescindible considerar lo que la investigación científica muestra respecto a los efectos que produce su consumo. El objetivo de esta revisión narrativa es revisar y discutir lo que ocasiona en el cerebro y sus consecuencias en la toma de decisiones y la inteligencia. Otras revisiones han mostrado que la atención, la memoria y la función psicomotora están afectadas en consumidores de mariguana (por ejemplo, ver Broyd, van Hell, Beale, Yücel, \& Solowij, 2015), pero poco se ha revisado sobre los efectos del consumo y la toma de decisiones y la inteligencia.

\section{MÉTODO}

A fin de obtener evidencia científica acerca de los efectos del consumo de mariguana en los cambios morfológicos cerebrales, y sobre la toma de decisiones y la inteligencia, se realizó una búsqueda, de acuerdo con
Ios criterios PRISMA (Moher, Liberati, Tetzlaff, \& Altman, 2009) en PubMed, en un periodo comprendido del $1 .{ }^{\circ}$ de enero de 2005 hasta el 10 de marzo de 2016 (al iniciar esta revisión), con la combinación de las siguientes palabras clave: (marijuana OR thc OR delta9-thc OR cannabis) AND (decision making OR lowa gambling task OR intelligence) (Figura 2). Se obtuvieron 566 artículos y se seleccionaron los que mostraban evidencia empírica de evaluación a sujetos humanos mediante la administración de pruebas cognitivas, en papel o computadora, los consumidores usaban cannabis como principal droga de uso, y podían o no evaluar la función cerebral. En el apartado de inteligencia, dado que ésta predice las situaciones importantes de la vida (Deary, 2012), como la educación, el ingreso, la ocupación, la salud, la enfermedad y la muerte, se incluyeron los resultados de un reporte en el que se relacionan la educación, el ingreso, la falta de ocupación y el uso de mariguana (Fergusson \& Boden, 2008), que estuvo entre los resultados de los artículos encontrados. Se excluyeron revisiones, estudios de caso y trabajos en los que los sujetos evaluados presentaran alguna psicopatología (excluyendo la dependencia de cannabis), condición neurológica, virus de inmunodeficiencia adquirida, dolor o cáncer; así como documentos que estuvieran en un idioma diferente al inglés.

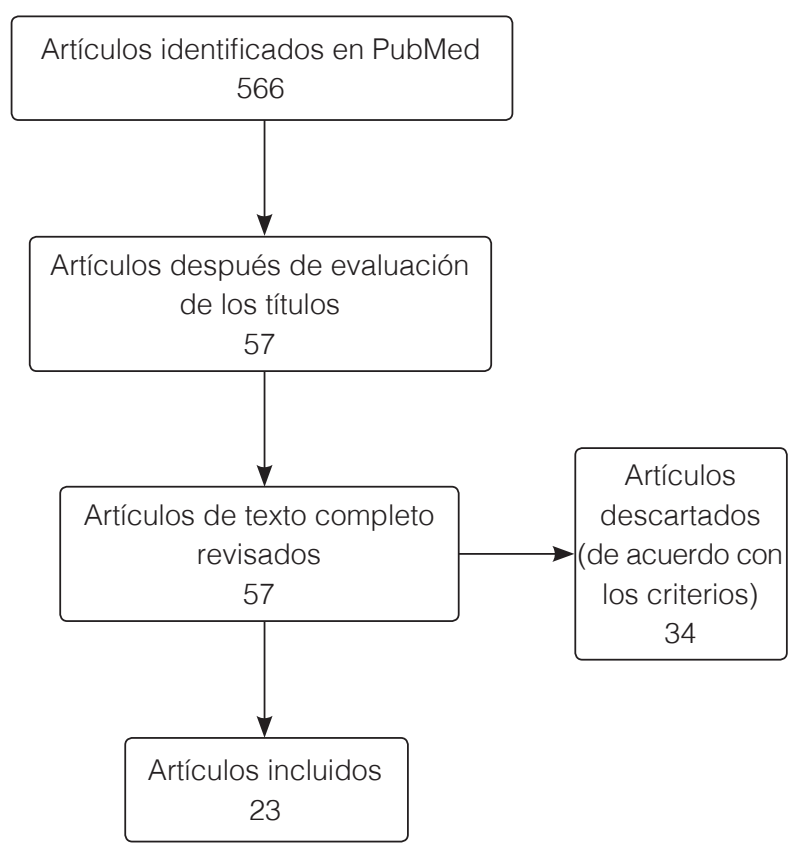

Figura 2. Diagrama de flujo de acuerdo con los criterios PRISMA para la identificación e inclusión de estudios en la presente revisión. Ver método para criterios de inclusión y exclusión 
Una dificultad asociada al estudio del efecto del consumo de mariguana es el hecho de que los usuarios son frecuentemente poliusuarios. Sin embargo, los estudios citados en esta revisión incluyen como covariable el consumo de otras drogas, legales e ilegales, para el análisis e interpretación de los resultados, así como el control de otras diversas variables. Así, los resultados descritos en esta revisión se pueden considerar como efecto del consumo de mariguana y no de otros potenciales factores.

\section{RESULTADOS}

\section{Consumo de mariguana y cambios morfológicos en el individuo}

Existen cambios morfológicos asociados con el consumo temprano de mariguana; por ejemplo, la talla (particularmente en hombres) y el peso durante la edad adulta son menores cuando el consumo se inició antes de los 17 años (Wilson, Mathew, Turkington, Hawk, Coleman, \& Provenzale, 2000). Además, existe una reducción en el volumen cerebral total y en el de la sustancia gris, mientras que también se observa un aumento en el volumen de la sustancia blanca (Wilson et al., 2000). Estos cambios morfológicos fueron más puntuales en regiones frontales y parietales. Estas estructuras participan en funciones como la atención (Uncapher, Hutchinson, \& Wagner, 2011), la memoria (Chadick \& Gazzaley, 2011) y la toma de decisiones (Blakemore \& Robbins, 2012).

Otras variables asociadas al consumo de mariguana se asocian con el volumen de diferentes regiones cerebrales; por ejemplo, el volumen de la amígdala y del hipocampo es inversamente proporcional al consumo de mariguana (Cousijn et al., 2012). También el volumen de la corteza orbitofrontal (COF, Figura 3) es inversamente proporcional a la edad de inicio del consumo de mariguana (Churchwell, López-Larson, \& Yurgelun-Todd, 2010). Estas tres regiones, amígdala, hipocampo y la COF, son de las más afectadas en la adicción (Koob \& Volkow, 2010). En particular, es de interés para esta revisión la COF, debido a su participación en la jerarquización del valor de los reforzadores y, por lo mismo, en la toma de decisiones (Tremblay \& Schultz, 1999).

Adicionalmente, se ha reportado una pérdida de la conectividad funcional cerebral (es decir, la correlación temporal de actividad entre dos regiones cerebrales) en reposo entre la corteza del cíngulo anterior (CCA) y el giro frontal superior, en adolescentes (Media, DE: 17, $\mathrm{DE}=2$ años) que tuvieron más de 50 exposiciones a mariguana y ésta era la droga de preferencia de consumo (edad de inicio: 13, DE=2.2 años), en comparación con sujetos no consumidores (Camchong, Lim, \& Kumra, 2016). Además, se mostró que 18 meses después de haberles detectado esta pérdida de la conectividad, los usuarios consumen cannabis mayor número de días (Camchong et al., 2016). Estos resultados sugieren que la mariguana, con este patrón de consumo, produce en los adolescentes un efecto dañino en la conectividad funcional en áreas relevantes para la toma de decisiones.

En usuarios "duros" de mariguana [28 $(\mathrm{DE}=8)$ años de edad, $10(\mathrm{DE}=6)$ cigarros de mariguana diarios; edad de inicio: 15 ( $D E=3$ ) años; duración de uso de cannabis: 12 $(\mathrm{DE}=7)$ años] el receptor $\mathrm{CB} 1$ presenta una disminución de alrededor del $20 \%$ en regiones corticales con respecto a sujetos no usuarios (Hirvonen et al., 2012). Otro estudio más reciente (Ceccarini et al., 2015) replicó estos resultados, no sólo en usuarios "duros", sino también en usuarios "moderados" (entre una y cuatro veces de uso a la semana) y de "bajo" uso (entre dos y tres veces al mes, o menos), que tenían características similares en edad (26, $\mathrm{DE}=4.1$ años), edad de inicio de consumo (15.8, $\mathrm{DE}=2.5$ años) y duración de uso de cannabis $(10.2, D E=4.6)$ al estudio de Hirvonen y colaboradores (Ceccarini et al., 2015). Dadas las similitudes entre los estudios, es posible sugerir que no parece ser la frecuencia de uso (que es la variable diferente entre los estudios) lo que explica la reducción del receptor CB1 en áreas corticales, sino que la edad de inicio y la duración del consumo están más fuertemente asociadas a la reducción del receptor. Es pertinente señalar que el receptor CB1 aumenta su expresión después de alrededor de cuatro semanas de abstinencia (Hirvonen et al., 2012), aunque no es claro si este aumento alcanza los niveles de los sujetos no usuarios. Con todo, merece la pena subrayar la reactividad del gen CNR1 ante estos estímulos.

Por otro lado, se ha reportado una variación en la expresión del ARN mensajero del receptor CB1 en el SNC, en función de la edad, lo que sugeriría un cambio en la expresión del receptor (Laprairie, Kelly, \& Denovan-Wright, 2012). Estos cambios incluyen a la corteza prefrontal dorsolateral (Figura 3), la cual también está involucrada en la toma de decisiones (Long, Lind, Webster, \& Weickert, 2012). La relevancia de estos cambios son en términos del desarrollo cerebral, ya que el receptor CB1 participa en la señalización para la diferenciación y la migración celular y, por lo mismo, para el establecimiento de redes neuronales (Gaffuri, Ladarre, \& Lenkei, 2012). En este sentido, se ha sugerido que el consumo de mariguana en etapas anteriores a completar la maduración cerebral modifica la conectividad neuronal en la edad adulta, haciéndola más difusa y menos eficiente (Zalesky et al., 2012). A pesar de que no podemos hablar de una relación causal entre los cambios morfológicos y los cambios en las funciones cognitivas, la probabilidad es alta. 

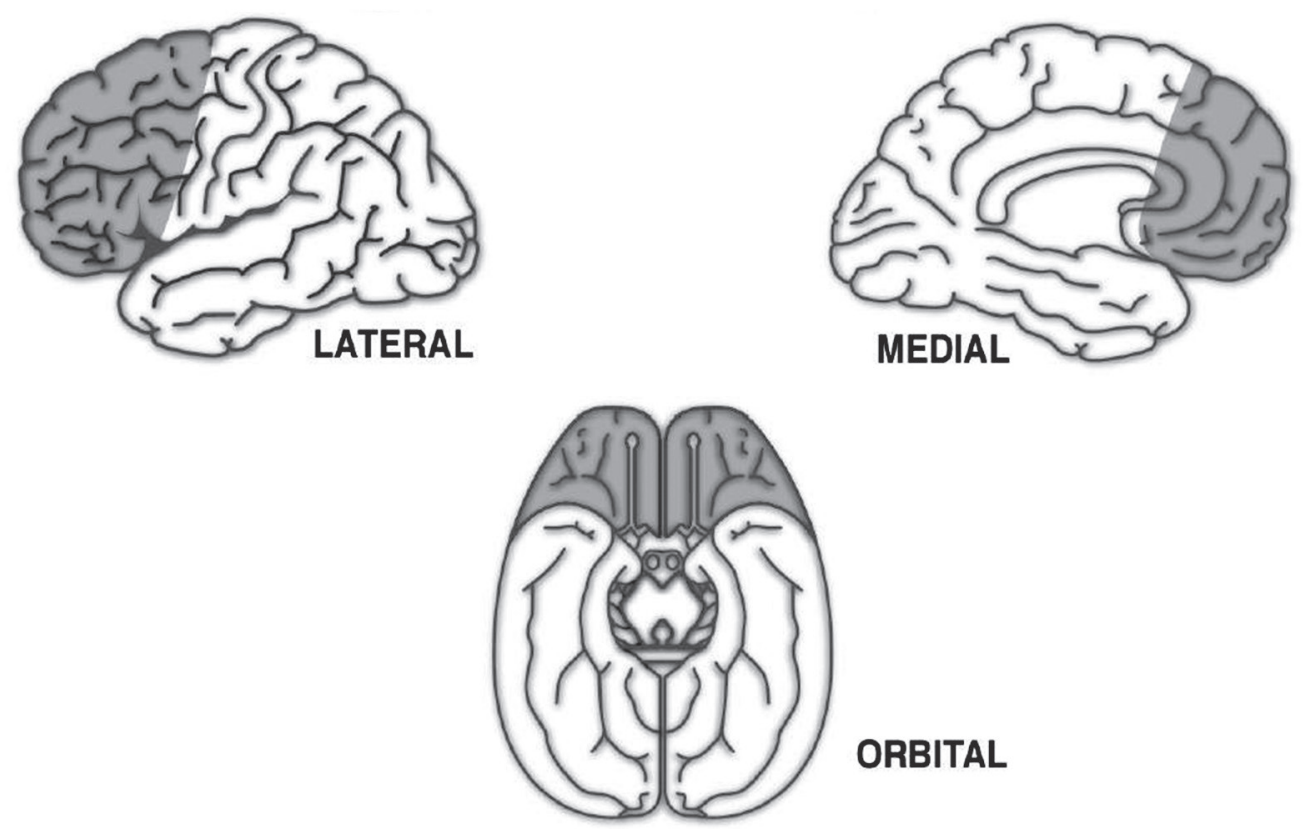

Figura 3. Ilustra de manera general las partes de la corteza prefrontal del humano. En la región que llamamos lateral se encuentra la corteza prefrontal dorsolateral, en la medial está la corteza prefrontal medial y ventral, mientras que en la que llamamos orbital, está la corteza prefrontal orbito frontal

\section{Consumo de mariguana y toma de decisiones}

Todos los días un sujeto enfrenta situaciones problemáticas que requieren que elija una estrategia para solucionarlas. La estrategia con la que decida contender con dichas situaciones tiene consecuencias que pueden ser las esperadas deseadas, las esperadas no deseadas o inesperadas, lo que impone un cierto grado de incertidumbre sobre el futuro del sujeto (Weller, Levin, \& Bechara, 2010). Por ello, lleva a cabo un proceso de evaluación de una variedad de opciones de respuesta y selecciona una, la que considere óptima, para resolver la situación, teniendo en cuenta las implicaciones o consecuencias en el futuro (Bechara, 2000; Clark, Cools, \& Robbins, 2004). A este proceso lo llamamos toma de decisiones.

Para evaluar conductualmente la toma de decisiones con frecuencia se emplea la tarea de apuesta de lowa (IGT, por sus siglas en inglés lowa Gambling Task), en la cual el sujeto utiliza unas barajas virtuales que le dan la posibilidad de ganar o perder dinero. En este paradigma, el sujeto asume una de dos estrategias: obtener fuertes ganancias, pero también grandes pérdidas (estrategia desfavorable), o tener ganancias y pérdidas moderadas (estrategia favorable). Los usuarios de mariguana tienden a emitir elecciones inmediatas (estrategia desfavorable) más que ventajosas (estrategia favorable) (Becker, Kerns, MacDonald, \& Carter, 2008; Bolla, Eldreth, Matochik, \& Cadet, 2005; Fridberg et al., 2010; Grant, Chamberlain, Schreiber, \& Odlaug, 2012; Wesley, Hanlon, \& Porrino, 2011). Además, se observa una menor activación en la red neuronal involucrada en la solución de la IGT, en comparación con los no usuarios: la CCA, la corteza frontal medial, la corteza parietal posterior, el lóbulo occipital y el cerebelo (Wesley et al., 2011). Para probar si después de 25 días de abstinencia se observaban cambios en la eficiencia conductual y en la actividad cerebral, se midió a consumidores "moderados" (8-35 cigarros por semana) y "duros" (53-84 cigarros por semana) de mariguana, además de no usuarios. Los usuarios "duros", a pesar de la abstinencia, siguieron ejecutando peor que los no usuarios; mientras que los "moderados" no difirieron en la eficiencia de los no usuarios, pero sí de los usuarios "duros". Por otro lado, éstos presentaron menor activación de la COF y mayor activación cerebelar, del cíngulo posterior y del giro lingual, que los usuarios "moderados". No se evaluaron diferencias contra el grupo de no usuarios (Bolla et al., 2005). Esto sugiere que 25 días de abstinencia son insuficientes para recuperar 
la eficiencia en la toma de decisiones cuando se tiene un consumo "duro" de mariguana. En los consumidores diarios de mariguana aumenta el tiempo que tardan en responder a la IGT, de manera dosis-dependiente, cuando están bajo intoxicación aguda con $\triangle 9$-THC, sin que se afecte la eficiencia para seleccionar las barajas ventajosas (Vadhan et al., 2007).

En usuarios adolescentes de mariguana (cuatro días a la semana) no se observan diferencias en la eficiencia durante la toma de decisiones en la IGT con respecto a los no usuarios (De Bellis et al., 2013; Dougherty et al., 2013), lo que sugiere que las alteraciones en la capacidad para tomar decisiones no es un déficit conductual en esta etapa. Sin embargo, en adolescentes que presentan dependencia de mariguana, a pesar de no diferir en la toma de decisiones con respecto a no usuarios, se observó una disminución en la actividad de la COF y un aumento en la activación de la corteza parietal, la corteza del cíngulo, el giro frontal y la ínsula, al efectuar elecciones riesgosas reforzantes, incluso con un periodo de abstinencia de $133.9 \pm 57.9$ días, en comparación con adolescentes no usuarios (De Bellis et al., 2013). La hiperactivación de dichas áreas supone un requerimiento mayor de recursos neuronales para mantener la misma ejecución conductual que los no usuarios; mientras que la hipoactivación de la COF se interpretó como una desregulación en la jerarquización de los estímulos reforzantes, lo que podría conferirles a los usuarios una vulnerabilidad al uso de sustancias, si esta hipoactividad hubiera existido antes del inicio del uso de mariguana.

Por otro lado, la frecuencia y el tiempo de consumo de mariguana modifican la ejecución en la capacidad para tomar decisiones. A mayor frecuencia y a mayor duración de uso de la sustancia, menor la capacidad para tomar decisiones correctas; es decir, la toma de decisiones en los usuarios de cannabis se hace menos lógicamente con la evidencia disponible conforme aumenta la frecuencia y la duración de uso de mariguana (Solowij et al., 2012). Esto sugeriría una falla en la flexibilidad cognitiva para adaptarse a los cambios impuestos por el ambiente, en comparación con los no usuarios (Lamers, Bechara, Rizzo, \& Ramaekers, 2006).

Asimismo, la activación de la corteza prefrontal ventromedial aumenta conforme mayor duración se tenga de consumir mariguana, sin importar la edad de inicio del consumo. Esta estructura está relacionada con la toma de decisiones emocionales y recibe una influencia directa del sistema de recompensa, en particular del núcleo accumbens y la amígdala. Por lo mismo, se ha asociado con la toma de decisiones de reforzadores en conflicto. Es decir, escoger entre uno bueno y otro mejor (Walton, Chau, \& Kennerley, 2015). A pesar de que los usuarios de mariguana presentan mayor activación de esta región, la toma de decisiones conductualmente no difiere de la de los no usuarios (Vaidya et al., 2012). Estos resultados sugieren que los consumidores crónicos de mariguana requieren de una mayor activación neuronal, tal vez como un efecto de compensación, a fin de tomar decisiones con la misma eficiencia que los no usuarios. Estos resultados son congruentes con los discutidos para la COF.

Todos estos resultados sugieren que el consumo de mariguana afecta los mecanismos cerebrales que modulan la toma de decisiones en condiciones de riesgo, reduciendo su eficiencia.

\section{Consumo de mariguana e inteligencia}

La inteligencia se puede definir como una capacidad general para razonar, resolver problemas, planear, pensar de manera abstracta, comprender y aprender de la experiencia (Gottfredson, 1997; Legg \& Hutter, 2007) o como la capacidad para generar un plan y las estrategias acordes para lograr eficaz y eficientemente un objetivo (Ruiz-Contreras \& Prospéro-García, 2009). Pruebas psicológicas permiten obtener el coeficiente intelectual (CI). El puntaje obtenido por el individuo se compara con normas de calificación para ubicarlo con respecto a un grupo. Se ha sugerido que el $\mathrm{Cl}$ predice el éxito en la vida académica (Gottfredson, 1998).

En un estudio longitudinal con una muestra de 1,037 individuos a quienes se les midió su $\mathrm{Cl}$ entre los 7 y los 13 años de edad y después a los 38 años, se observó una caída significativa de seis puntos en el $\mathrm{Cl}$ de los individuos que reportaron dependencia de mariguana, con respecto a la medición inicial (Meier et al., 2012). Esta caída en el $\mathrm{Cl}$ no se explica por otras variables como dependencia o consumo de otras sustancias o uso reciente o no de mariguana, o padecer esquizofrenia o el estatus socioeconómico. La caída del Cl sólo se observó en los sujetos que fueron diagnosticados con al menos tres veces dependencia de mariguana antes de los 18 años. Pero no para los que fueron diagnosticados después de esta edad (Meier et al., 2012). Este estudio revela que la caída en la inteligencia está fuertemente asociada a la edad de inicio y el patrón de consumo de mariguana.

El consumo de mariguana entonces es una variable que predice deterioro de la inteligencia. Un mayor número de días que un adolescente se exponga al uso de esta sustancia se asocia con una reducción en el Cl y con un enlentecimiento en la velocidad de procesamiento durante la evaluación de atención (Camchong et al., 2016). Asimismo, adolescentes consumidores de mariguana presentan menor aprovechamiento escolar (por ejemplo, fluidez verbal y matemática, cálculo, comprensión de lectura) que adolescentes no usuarios (Brook, 
Stimmel, Zhang, \& Brook, 2008; Hooper, Woolley, \& De Bellis, 2014); el inicio de dependencia de cannabis, la edad de inicio regular y la frecuencia de uso se correlacionaron positivamente con el aprovechamiento (Hooper et al., 2014). En una cohorte de adolescentes, a quienes se evaluó su Cl entre los 9 y los 10 años y se les volvió a evaluar 10 años después, se mostró una caída significativa en el mismo de cuatro puntos en aquellos individuos que consumieron más de 30 veces mariguana, aunque esta caída se limitó a las habilidades verbales, pero no a las espaciales (Jackson et al., 2016). Estos resultados sugieren un deterioro en la organización funcional asociada a información verbal versus espacial que se observa en la edad adulta temprana (19 años de edad).

El impacto del consumo de mariguana sobre la inteligencia puede valorarse con resultados de otros estudios en los que se miden variables que evalúan éxito en la vida (Fergusson \& Boden, 2008). Por ejemplo, mientras mayor haya sido el consumo de mariguana (de cero a 400 veces o más) entre los 14 y los 21 años de edad, la probabilidad de dejar inconclusos los estudios universitarios es mayor. Asimismo, perciben un menor ingreso anual, tienen mayor riesgo a estar desempleados y de vivir de pensión gubernamental, así como de tener una menor satisfacción en la vida, en particular en las relaciones interpersonales (Fergusson \& Boden, 2008). Estos resultados sugieren que el consumo "duro" de mariguana en la adolescencia y en la edad adulta temprana predice resultados desfavorables en variables asociadas al éxito en la vida. Y si ser más inteligente predice mayor éxito en la vida y tener dependencia de mariguana aumenta el riesgo de reducirla, lo esperado es que el usuario contienda con menos recursos las demandas diarias y por lo mismo con menos éxito.

\section{DISCUSIÓN Y CONCLUSIONES}

Una de las variables que parece potenciar los efectos adversos del consumo de mariguana es la edad de inicio. Mientras más joven se empiece a consumir mariguana, mayor es el riesgo de modificar la conectividad neuronal y, consecuentemente, afectar el funcionamiento cerebral involucrado en nuestra capacidad de toma de decisiones y de nuestra inteligencia. Estos efectos influyen sobre la manera en la que las personas se adaptan a la vida cotidiana, por lo que puede tener impacto en el ingreso económico y en la satisfacción con el estilo de vida que se tenga. Variables como la frecuencia de uso y la cantidad de consumo de mariguana afectan también la toma de decisiones. Esta información es relevante para el sector salud, con el fin de crear campañas que eviten su consumo, así como para llevar a cabo acciones para facilitar la rehabilitación.
Sin embargo, falta conocer los potenciales efectos que tenga el consumo temprano, frecuente y "duro" de mariguana en otros periodos de la vida de los individuos, así como conocer si esta sustancia participa en el desarrollo de patologías como depresión, ansiedad e incluso demencia en los usuarios.

Por último, la mariguana y sus efectos están de alguna manera revelando las diversas funciones cerebrales en las que el sistema endocannabinoide participa, mostrándonos su importancia al menos en las dos esferas conductuales discutidas en esta revisión: la toma de decisiones y la inteligencia.

\section{FUENTES DE FINANCIAMIENTO}

Este trabajo fue apoyado por los proyectos: IN223314 a AERC, IN218316 a OPG e IA2071416 a MMD de la Dirección General de Asuntos del Personal Académico de la Universidad Nacional Autónoma de México.

\section{CONFLICTOS DE INTERÉS}

Los autores no presentan conflicto de interés.

\section{REFERENCIAS}

American Psychiatric Association. (2013). Diagnostic and Statistical Manual of Mental Disorders: DSM-5. (5ta. edición). Washington, DC: Autor.

Ashton, C. H. (2001). Pharmacology and effects of cannabis: A brief review. British Journal of Psychiatry, 178(2), 101-106. doi:10.1192/bjp.178.2.101

Bechara, A. (2000). Characterization of the decision-making deficit of patients with ventromedial prefrontal cortex lesions. Brain, 123(11), 2189-2202. doi: 10.1093/brain/123.11.2189

Becker, T. M., Kerns, J. G., MacDonald, A. W., \& Carter, C. S. (2008). Prefrontal dysfunction in first-degree relatives of schizophrenia patients during a stroop task. Neuropsychopharmacology, 33(11), 2619-2625. doi: 10.1038/sj.npp.1301673

Blakemore, S. J., \& Robbins, T. W. (2012). Decision-making in the adolescent brain. Nature Neuroscience, 15(9), 1184-1191. doi: 10.1038/nn.3177

Bolla, K. I., Eldreth, D. A., Matochik, J. A., \& Cadet, J. L. (2005). Neural substrates of faulty decision-making in abstinent marijuana users. Neurolmage, 26(2), 480-492. doi: 10.1016/j.neuroimage.2005.02.012

Bossong, M. G., Jansma, J. M., van Hell, H. H., Jager, G., Oudman, E., Saliasi, E., ... Ramsey, N. F. (2012). Effects of $\Delta$ 9-tetrahydrocannabinol on human working memory function. Biological Psychiatry, 71(8), 693-699. doi: 10.1016/j.biopsych.2012.01.008

Brook, J. S., Stimmel, M. A., Zhang, C., \& Brook, D. W. (2008). The association between earlier marijuana use and subsequent academic achievement and health problems: A longitudinal study. American Journal on Addictions, 17(2), 155-160. 
Broyd, S. J., van Hell, H. H., Beale, C., Yücel, M., \& Solowij, N. (2015). Acute and chronic effects of cannabinoids on human cognition. A systematic review. Biological Psychiatry, 79(7), 557-67. doi: 10.1016/j.biopsych.2015.12.002

Brunet, B., Doucet, C., Venisse, N., Hauet, T., Hébrard, W., Papet, Y., ... Mura, P. (2006). Validation of large white pig as an animal model for the study of cannabinoids metabolism: Application to the study of THC distribution in tissues. Forensic Science International, 161(2-3), 169-174. doi: org/10.1016/j.forsciint.2006.04.018

Burns, H., Van Laere, K., Sanabria-Bohórquez, S., Hamill, T. G., Bormans, G., Eng, W., ... Hargreaves, R. J. (2007). [18F]MK-9470, a positron emission tomography (PET) tracer for in vivo human PET brain imaging of the cannabinoid-1 receptor. Proceedings of the National Academy of Sciences of the United States of America, 104, 9800-9805.

Camchong, J., Lim, K. O., \& Kumra, S. (2016). Adverse effects of cannabis on adolescent brain development: A longitudinal study. Cerebral Cortex, 1-9. doi: 10.1093/cercor/bhw015

Ceccarini, J., Kuepper, R., Kemels, D., Van Os, J., Henquet, C., \& Van Laere, K. (2015). [18F]MK-9470 PET measurement of cannabinoid $\mathrm{CB} 1$ receptor availability in chronic cannabis users. Addiction Biology, 20(2), 357-367. doi: 10.1111/adb.12116

Chadick, J. Z., \& Gazzaley, A. (2011). Differential coupling of visual cortex with default or frontal-parietal network based on goals. Nature Neuroscience, 14(7), 830-832. doi: 10.1038/nn.2823

Churchwell, J. C., López-Larson, M., \& Yurgelun-Todd, D. A. (2010). Altered frontal cortical volume and decision making in adolescent cannabis users. Frontiers in Psychology, 1, 1-8. doi:10.3389/fpsyg.2010.00225

Clark, L., Cools, R., \& Robbins, T. W. (2004). The neuropsychology of ventral prefrontal cortex: decision-making and reversal learning. Brain and Cognition, 55(1), 41-53. doi:10.1016/S02782626(03)00284-7

Cousijn, J., Wiers, R. W., Ridderinkhof, K. R., Van den Brink, W., Veltman, D. J., \& Goudriaan, A. E. (2012). Grey matter alterations associated with cannabis use: Results of a VBM study in heavy cannabis users and healthy controls. Neurolmage, 59(4), 38453851. doi: 10.1016/j.neuroimage.2011.09.046

Deary, I. J. (2012). Intelligence. Annual Review of Psychology, 63, 453-482.

De Bellis, M. D., Wang, L., Bergman, S. R., Yaxley, R. H., Hooper, R., \& Huettel, S. A. (2013). Neural mechanisms of risky decision-making and reward response in adolescent onset cannabis use disorder. Drug and Alcohol Dependence, 133(1), 134-145. doi: 10.1016/j.drugalcdep.2013.05.020

D'Souza, D. C., Fridberg, D. J., Skosnik, P. D., Williams, A., Roach, B., Singh, N., ... Mathalon, D. (2012). Dose-related modulation of event-related potentials to novel and target stimuli by intravenous $\triangle 9-T H C$ in humans. Neuropsychopharmacology, 37(7), 1632-1646. doi:10.1038/npp.2012.8

Dougherty, D. M., Mathias, C. W., Dawes, M. A., Furr, R. M., Charles, N., Liguori, A., ... Acheson, A. (2013). Impulsivity, attention, memory, and decision-making among adolescent marijuana users.
Psychopharmacology, 226(2), 307-319. doi: 10.1007/s00213012-2908-5

Felder, C. C., Nielsen, A., Briley, E. M., Palkovits, M., Priller, J., Axelrod, J., ... Becker, G. W. (1996). Isolation and measurement of the endogenous cannabinoid receptor agonist, anandamide, in brain and peripheral tissues of human and rat. FEBS Letters, 393(2-3), 231-235. doi:10.1016/0014-5793(96)00891-5

Fergusson, D. M., \& Boden, J. M. (2008). Cannabis use and later life outcomes. Addiction, 103(6), 969-976. doi: 10.1111/j.13600443.2008.02221.x

Fridberg, D. J., Queller, S., Ahn, W. Y., Kim, W., Bishara, A. J., Busemeyer, J. R., ... Stout, J. C. (2010). Cognitive mechanisms underlying risky decision-making in chronic cannabis users. Journal of Mathematical Psychology, 54(1), 28-38. doi: 10.1016/j. jmp.2009.10.002

Gaffuri, A. L., Ladarre, D., \& Lenkei, Z. (2012). Type-1 cannabinoid receptor signaling in neuronal development. Pharmacology, 90(12), 19-39. doi: 10.1159/000339075

Giuffrida, A., Leweke, F. M., Gerth, C. W., Schreiber, D., Koethe, D., Faulhaber, J., ... Piomelli, D. (2004). Cerebrospinal anandamide levels are elevated in acute schizophrenia and are inversely correlated with psychotic symptoms. Neuropsychopharmacology, 29(11), 2108-2114. doi: 10.1038/sj.npp.1300558

Gottfredson, L. (1998). The general intelligence factor. Scientific American Presents, 9(4), 24-29.

Gottfredson, L. S. (1997). Mainstream science on intelligence: An editorial with 52 signatories, history, and bibliography. Intelligence, 24(1), 13-23. doi: 10.1016/S0160-2896(97)90011-8

Grant, J. E., Chamberlain, S. R., Schreiber, L., \& Odlaug, B. L. (2012). Neuropsychological deficits associated with cannabis use in young adults. Drug and Alcohol Dependence, 121(1), 159-162.

Haney, M., Ward, A. S., Comer, S. D., Foltin, R. W., \& Fischman, M. W. (1999a). Abstinence symptoms following oral THC administration to humans. Psychopharmacology, 141(4), 385-394. doi: $10.1007 / \mathrm{s} 002130050848$

Haney, M., Ward, A. S., Comer, S. D., Foltin, R. W., \& Fischman, M. W. (1999b). Abstinence symptoms following smoked marijuana in humans. Psychopharmacology, 141(4), 395-404. doi:10.1007/ s002130050849

Hart, C. L., Van Gorp, W., Haney, M., Foltin, R. W., \& Fischman, M. W. (2001). Effects of acute smoked marijuana on complex cognitive performance. Neuropsychopharmacology, 25(5), 757-765.

Herkenman, M., Lynn, A. B., Johnson, M. R., Melvin, L. S., de Costa, B. R., \& Rice, C. (1991). Characterization and localization of cannabinoid receptors in rat brain: a quantitative in vitro autoradiographic study. The Journal of Neuroscience, 11(2), 563-583.

Herkenham, M., Lynn, A. B., Little, M. D., Johnson, M. R., Melvin, L. S., de Costa, B. R., ... Rice, K. C. (1990). Cannabinoid receptor localization in brain. Neurobiology, 87(5), 1932-1936.

Herrera-Solís, A., Vásquez, K. G., \& Prospéro-García, O. (2010). Acute and subchronic administration of anandamide or oleamide increases REM sleep in rats. Pharmacology Biochemistry and Behavior, 95(1), 106-112. doi: 10.1016/j.pbb.2009.12.014 
Hirvonen, J., Goodwin, R. S., Li, C. T., Terry, G. E., Zoghbi, S. S., Morse, C., ... Innis, B.R. (2012). Reversible and regionally selective downregulation of brain cannabinoid CB1 receptors in chronic daily cannabis smokers. Molecular Psychiatry, 17(6), 642-649. doi: $10.1038 / \mathrm{mp} .2011 .82$

Hooper, S. R., Woolley, D., \& De Bellis, M. D. (2014). Intellectual, neurocognitive, and academic achievement in abstinent adolescents with cannabis use disorder. Psychopharmacology, 231(8), 1467-1477.

Howlett, A. C., Barth, F., Bonner, T. I., Cabral, G., Casellas, P., Devane, W. A., ... Pertwee, R. G. (2002). International Union of Pharmacology. XXVII. Classification of cannabinoid receptors. Pharmacological Reviews, 54(2), 161-202.

Huestis, M. A. (2005). Pharmacokinetics and metabolism of the plant cannabinoids, d9-tetrahydrocannabinol, cannabidiol and cannabinol. En Pertwee, R. (Ed.). Handbook of Experimental Pharmacology (Vol. 168, pp. 657-690). Berlin: Springer-Verlag.

Jackson, N. J., Isen, J. D., Khoddam, R., Irons, D., Tuvblad, C., Iacono, W. G., ... Baker, L. A. (2016). Impact of adolescent marijuana use on intelligence: Results from two longitudinal twin studies. Proceedings of the National Academy of Sciences, 113(5), E500-E508. doi: 10.1073/pnas. 1516648113

Kirkham, T. C., Williams, C. M., Fezza, F., \& Di Marzo, V. (2002). Endocannabinoid levels in rat limbic forebrain and hypothalamus in relation to fasting, feeding and satiation: stimulation of eating by 2-arachidonoyl glycerol. British Journal of Pharmacology, 136(4), 550-557. doi: 10.1038/sj.bjp.0704767

Koob, G. F., \& Volkow, N. D. (2010). Neurocircuitry of addiction. Neuropsychopharmacology, 35(1), 217-238. doi:10.1038/ npp.2009.110

Lamers, C. T. J., Bechara, A., Rizzo, M., \& Ramaekers, J.G. (2006). Cognitive function and mood in MDMA/THC users, THC users and non-drug using controls. Journal of Psychopharmacology, 20(2), 302-311.

Laprairie, R. B., Kelly, M. E. M., \& Denovan-Wright, E. M. (2012). The dynamic nature of type 1 cannabinoid receptor (CB1) gene transcription. British Journal of Pharmacology, 167(8), 15831595. doi.org/10.1111/j.1476-5381.2012.02175.x

Legg, S., \& Hutter, M. (2007). A collection of definitions of intelligence. Frontiers in Artificial Intelligence and applications, 157, 17-24.

Long, L. E., Lind, J., Webster, M., \& Weickert, C. S. (2012). Developmental trajectory of the endocannabinoid system in human dorsolateral prefrontal cortex. BMC Neuroscience, 13(87). doi: 10.1186/1471-2202-13-87

Meier, M. H., Caspi, A., Ambler, A., Harrington, H., Houts, R., Keefe, R. S. E., ... Moffitt, T. E. (2012). Persistent cannabis users show neuropsychological decline from childhood to midlife. Proceedings of the National Academy of Sciences, 109(40), E2657-E2664. doi: 10.1073/pnas.1206820109

Mendelson, W. B. \& Basile, A. (1999) The hypnotic actions of oleamide are blocked by a camabinoid receptor antagonist. Neuroreport, 10(15), 3237-3239
Méndez-Díaz, M., Caynas-Rojas, S., Arteaga Santacruz, V., Ruiz-Contreras, A. E., Aguilar-Roblero, R., \& Prospéro-García, O. (2013). Entopeduncular nucleus endocannabinoid system modulates sleep-waking cycle and mood in rats. Pharmacology Biochemistry and Behavior, 107, 29-35. doi: 10.1016/j. pbb.2013.04.003

Méndez-Díaz, M., Herrera-Solís, A., Soria-Gómez, E., Rueda-Orozco, P., \& Prospéro-García, O. (2008). Mighty cannabinoids: A potential pharmacological tool in medicine. En M. Méndez \& R. Mondragón-Ceballos (Eds.), Neural Mechanisms of Action of Drugs of Abuse and Natural Reinforcers (Vol. 661, pp. 137-157). Kerala, India: Research Signpost.

Méndez-Díaz, M., Rueda-Orozco, P. E., Ruiz-Contreras, A. E., \& Prospéro-García, O. (2012). The endocannabinoid system modulates the valence of the emotion associated to food ingestion. Addiction Biology, 17(4), 725-735. doi: 10.1111/j.13691600.2010.00271.x

Moher, D., Liberati, A., Tetzlaff, J., \& Altman, D. G. (2009). Preferred reporting items for systematic reviews and meta-analyses: The PRISMA Statement. PLoS Med. 6:e1000097.

Oficina de las Naciones Unidas contra la Droga y el Delito. (2015) World Drug Report. Viena: Autor. Recuperado de https://www. unodc.org/documents/wdr2015/World_Drug_Report_2015.pdf

Pedroza-Llinás, R., Méndez-Díaz, M., Ruiz-Contreras, A. E., \& Prospéro-García, O. (2013). CB1 receptor activation in the nucleus accumbens core impairs contextual fear learning. Behavioural Brain Research, 237, 141-147. doi: 10.1016/j.bbr.2012.09.032

Romano-López, A., Méndez-Díaz, M., Ruiz-Contreras, A. E., Carrisoza, R., \& Prospéro-García, O. (2012). Maternal separation and proclivity for ethanol intake: A potential role of the endocannabinoid system in rats. Neuroscience, 223, 296-304. doi: 10.1016/j. neuroscience.2012.07.071

Ruiz-Contreras, A. E., Carrillo-Sánchez, K., Ortega-Mora, I., Barrera-Tlapa, M. A., Román-López, T. V., Rosas-Escobar, C. B., ... Prospéro-García, O. (2014). Performance in working memory and attentional control is associated with the rs2180619 SNP in the CNR1 gene. Genes, Brain and Behavior, 13(2), 173-178. doi: 10.1111/gbb.12097

Ruiz-Contreras, A., Delgado-Herrera, M., García-Vaca, P., Almeida-Rosas, G. A., Soria-Rodríguez, G., Soriano-Bautista, A., .. Prospéro-García, O. (2011). Involvement of the AATn polymorphism of the CNR1 gene in the efficiency of procedural learning in humans. Neuroscience Letters, 494(3), 202-206.

Ruiz-Contreras, A. E., \& Prospéro-García, O. (2009) Los genios, los otros y sus genes. En E. A. Escotto-Córdova \& M. A. Villa, (Eds.), Desarrollo y alteraciones del lenguaje, neuropsicología y genética de la Inteligencia (pp. 275-304). México: Facultad de Estudios Superiores Zaragoza, UNAM

Solowij, N., Jones, K. A., Rozman, M. E., Davis, S. M., Ciarrochi, J., Heaven, P. C. L., ... Yücel, M. (2012). Reflection impulsivity in adolescent cannabis users: A comparison with alcohol-using and non-substance-using adolescents. Psychopharmacology 219(2), 575-586. doi: 10.1007/s00213-011-2486-y 
Soria-Gómez, E., Matías, I., Rueda-Orozco, P. E., Cisneros, M., Petrosino, S., Navarro, L., ... Prospéro-García, O. (2007). Pharmacological enhancement of the endocannabinoid system in the nucleus accumbens shell stimulates food intake and increases c-Fos expression in the hypothalamus. British Journal of Pharmacology, 151(7), 1109-1116. doi: 10.1038/sj.bjp.0707313

Tremblay, L., \& Schultz, W. (1999). Relative reward preference in primate orbitofrontal cortex. Nature, 398(6729), 704-708. doi: $10.1038 / 19525$

Uncapher, M. R., Hutchinson, J. B., \& Wagner, A. D. (2011). Dissociable effects of top-down and bottom-up attention during episodic encoding. Journal of Neuroscience, 31(35), 12613-12628. doi: 10.1523/JNEUROSCI.0152-11.2011

Vadhan, N. P., Hart, C. L., van Gorp, W. G., Gunderson, E. W., Haney, M., \& Foltin, R. W. (2007). Acute effects of smoked marijuana on decision making, as assessed by a modified gambling task, in experienced marijuana users. Journal of Clinical and Experimental Neuropsychology, 29(4), 357-364. doi: 10.1080/13803390600693615

Vaidya, J. C., Block, R. I., O'Leary, D. S., Ponto, L. B., Ghoneim, M. M., \& Bechara, A. (2012). Effects of chronic marijuana use on brain activity during monetary decision-making. Neuropsychopharmacology, 37, 618-629. doi: 10.1038/npp.2011.227
Walton, M. E., Chau, B. K. H., \& Kennerley, S. W. (2015). Prioritising the relevant information for learning and decision making within orbital and ventromedial prefrontal cortex. Current Opinion in Behavioral Sciences, 1, 78-85. doi: 10.1016/j.cobeha.2014.10.005 Weller, J. A, Levin, I. P., \& Bechara, A. (2010). Do individual differences in lowa Gambling Task performance predict adaptive decision making for risky gains and losses? Journal of Clinical and Experimental Neuropsychology, 32(2), 141-150. doi: 10.1080/13803390902881926

Wesley, M. J., Hanlon, C. A., \& Porrino, L. J. (2011). Poor decision-making by chronic marijuana users is associated with decreased functional responsiveness to negative consequences. Psychiatry Research Neuroimaging, 191(1), 51-59. doi: 10.1016/j.pscychresns.2010.10.002

Wilson, W., Mathew, R., Turkington, T., Hawk, T., Coleman, E., \& Provenzale, J. (2000). Brain morphological changes and early marijuana use: A magnetic resonance and positron emission tomography study. Journal of Addictive Diseases, 19(1), 1-22. doi: 10.1300/J069v19n01_01

Zalesky, A., Solowij, N., Yücel, M., Lubman, D. I., Takagi, M., Harding, I. H., ... Seal, M. (2012). Effect of long-term cannabis use on axonal fibre connectivity. Brain, 135(7), 2245-2255. doi: 10.1093/brain/aws136 